\title{
The Design of a Novel Measurement System for the Characterization of Gas Sensing Materials
}

\author{
Xijin Leng ${ }^{1}$, Bangning Sun ${ }^{2}$, Guolong Liu $^{3}$, Hui Suo ${ }^{* 4, \text { a }}$ \\ 1,2,3,4 College of Electronic Science and Engineering, Jilin University, Changchun, 130012, China \\ âE-mail: suohui@jlu.edu.cn
}

Keywords: Novel Gas Sensitive Test System; Brittle Material Test; Step Type Heating; PID Control

\begin{abstract}
A novel measurement system for gas sensing materials is designed, and is suitable for sheet shaped or unpacked materials. Except for protecting powder materials during test procedures, this system complements the protection of brittle materials which is increasingly popular. The system has two working modes: multistep elevated temperature and constant temperature control, applied in the pre testing phase of the material and response \& recovery characteristics test phase respectively. The two working modes match with different test circuits and could be selected by the software of the system. The temperature control is realized by the combination of conventional PID control and fuzzy PID control, controlling temperature fast and accurately. These features make it a convenient and efficient system for determining the principal performance indexes of a gas sensing material (e.g. optimum operating temperature point, sensitivity, stability, selectivity, response/recovery times, etc.).
\end{abstract}

\section{Introduction}

In response to the needs for measuring gas concentrations in many different fields of application, from the food quality assessment, to the combustion process controlling and monitoring, a lot of attention has been paid to the development of solid state gas sensors [1]. The powdered metal oxide semiconductor and corresponding testing instrument [2] [3] [4] have been studied for more than 40 years but most of the test aims for powder or film materials, and the temperature of the measurement is predicted. It must be stressed that the sensor performance is strongly dependent on its working conditions, like temperature and humidity [5] [6]. Therefore, the temperature of gas sensitive materials should be accurately measured and controlled.

The bender-free method of growing gas sensing materials on foam metal has attracts a great attention for the past few years. Devices manufactured by binder-free technology could gain a longer cycle life and a lower internal resistance, providing a better cost performance. Notwithstanding remarkable progress in gas sensor with binder-free technology, some challenges still remain hindering application on a very large scale commensurate with global demand. One of the most serious challenges is the gas-sensitive test of sheet samples with brittle structure caused by the imperative high temperature sintering procedure. Conventional testing instruments could easily break the samples by squeeze it badly. Aiming to address this challenge, new test system with special protection of brittle samples thereby becomes particularly indispensable.

In this work, a novel measurement system for gas sensing materials is presented. The system is suitable for not only powder materials but also flake brittle materials especially benefit the tests of in-situ growth gas sensing materials. The temperature of the material can be measured accurately during gas-sensitive testing. The system has two working modes: multistep elevated temperature (named mode one) and constant temperature control (named mode two). They are applied in the pre testing phase of the material and response \& recovery characteristics test phase respectively. These features make the system suitable for determining the principal performance indexes of a gas sensing material and improve the efficiency of study of gas sensing materials. 


\section{System Architecture}

\section{A. Test Position Overview}

In order to realize adjustment of the contact degree to protect the gas sensitive material, the test platform is designed (Fig. 1). The main body part of the test platform is a microscope lift bracket. From the bottom up, the test platform consists of a micromatic setting, a ceramic heating sheet and the two test probes over the heating ceramic sheet. Spring pressure of the test probes is 19 20g and the height conditioning of the test probes can be carried out by ascending and descending the support of the microscope. The accuracy is $1 \mathrm{~mm}$ and the adjusting range is $80 \mathrm{~mm}$. Micromatic setting can be adjusted in the XYZ direction. Adjusting precision is $0.1 \mathrm{~mm}$ and adjusting distance is $20 \mathrm{~mm}$.

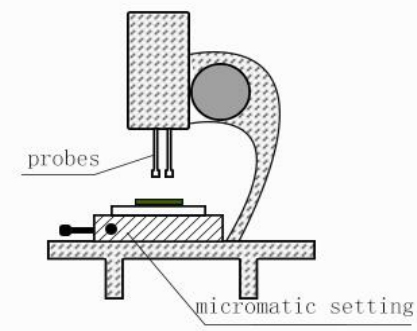

Fig. 1. Test platform overview

\section{B. Measurement System Frame}

The measurement system frame is shown in Figure 2 including resistance measurement and temperature measurement and control. The measurement system has two ARMs. ARM I is responsible for the signal processing of the resistance, LCD display, and communicates with PC and exchanges information with ARM II (including reading temperature, sending the expected temperature value, etc.). ARM II is mainly responsible for the measurement of temperature by sending the real-time temperature value to ARM I , and controlling temperature using PID control, according to the temperature ARM I set.

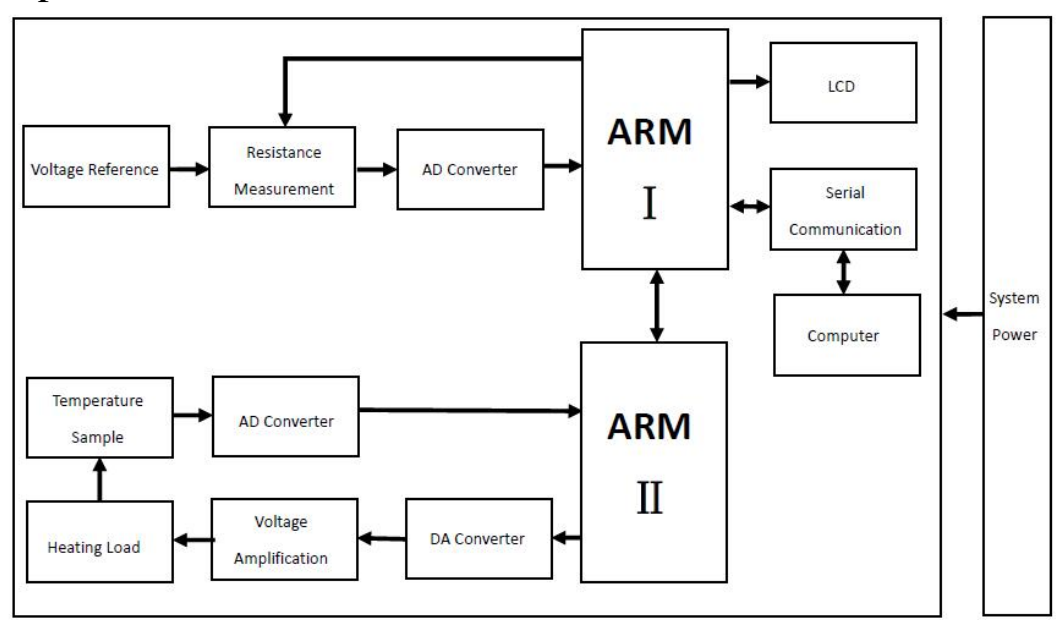

Fig. 2. Measurement system frame

\section{Temperature Measurement and Control System}

$\mathrm{K}$ type thermocouple, a common temperature sensor, is used to measure temperature. The measurement range is $0 \sim 1300{ }^{\circ} \mathrm{C}$. Temperature AD conversion is realized by MAX31855, which is produced by the Maxim Integrated. The chip is designed specifically for the thermocouple. The MAX31855 is a sophisticated thermocouple-to-digital converter with a built-in 14-bit analog-to-digital converter (ADC). The device also contains cold-junction compensation sensing and correction. For a K-type thermocouple, the voltage changes by about $41 \mu \mathrm{V} /{ }^{\circ} \mathrm{C}$.

Fig. 3 shows the measurement circuit. The resolution of the system is $0.1^{\circ} \mathrm{C}$, and the error rate is less than $1 \%$ after nonlinear correction. 
Generally, it is difficult to get a precise mathematical model of the object. Conventional PID control algorithm cannot give consideration to both fast and stable [7] [8]. The fuzzy outputs of 3 control coefficients of PID are divided into 7 Fuzzy States and the control rules of fuzzy controller summed up by experience can make the temperature more stable. Therefore, the temperature control of the system is divided into fuzzy PID control area and direct control area. The fuzzy PID control algorithm is used to stabilize the temperature in a certain error range which is close to the target temperature. When the temperature exceeds the above error range, the direct control algorithm ensures the speed of heating up achieving fast and stable results.

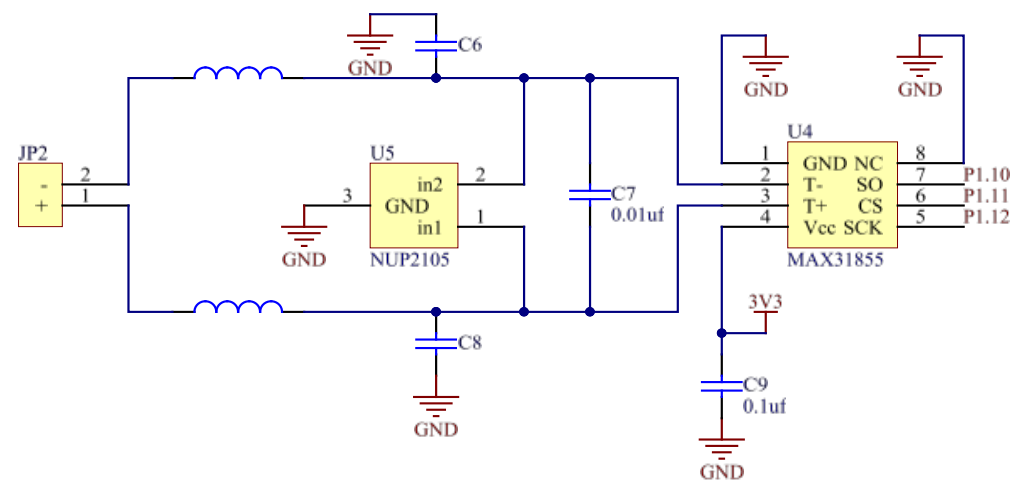

Fig. 3. Temperature measurement circuit

\section{Resistance Measurement}

Simplified circuit diagram of resistance measurement shows in Fig. 4. In this system, Vref is kept stable 5V. Three precision resistors Rref with the same resistance and gas sensitive material $\mathrm{Rx}$ make up a resistance bridge. However, the potential range of the signal acquired from the resistance bridge is $-2.5 \mathrm{~V}$ to $2.5 \mathrm{~V}$ and hardly received by $\mathrm{AD}$. The $\mathrm{AD} 620$ is used to solve this problem by boosting the minimum potential of the signal from -2.5 volts to 0 volts which is suitable for the AD. Furthermore, a TLV2252 was used as voltage follower to improve the load capacity. The output voltage can be written as:

$\mathrm{Vo}=2.5 \mathrm{~V}+\left(\frac{\text { Vref }}{R r e f+R x} * R x-\frac{\text { Vref }}{2 R r e f} *\right.$ Rref $)$

$V_{0} * R r e f+V_{0} * R x=5 V * R x$

The unknown resistance can be written as follow.

$R x=\frac{V_{0}}{5 V-V_{o}} *$ Rref

We use MAX144, 12-bit analog-to-digital converters (ADCs) as the resistance measurement circuit of AD conversion. The system has 6 similar resistance bridges and the Rref value is $100 \Omega$, $1 \mathrm{~K} \Omega, 10 \mathrm{~K} \Omega, 100 \mathrm{k} \Omega, 1 \mathrm{M} \Omega$, and $10 \mathrm{M} \Omega$ with the corresponding measurement range of $1 \Omega \sim 10 \mathrm{k} \Omega$, $10 \Omega \sim 100 \mathrm{k} \Omega, 100 \Omega \sim 1 \mathrm{M} \Omega, 1 \mathrm{~K} \Omega \sim 10 \mathrm{M} \Omega, 10 \mathrm{~K} \Omega \sim 100 \mathrm{M} \Omega$, and $100 \mathrm{k} \Omega \sim 1 \mathrm{G} \Omega$, respectively. The measurement range can cover all the sensitivity of the gas sensitive material below 10000 . Measuring speed can reach 10 times/s even above $1 \mathrm{M} \Omega$. The system comprehensive error is $2 \%$.

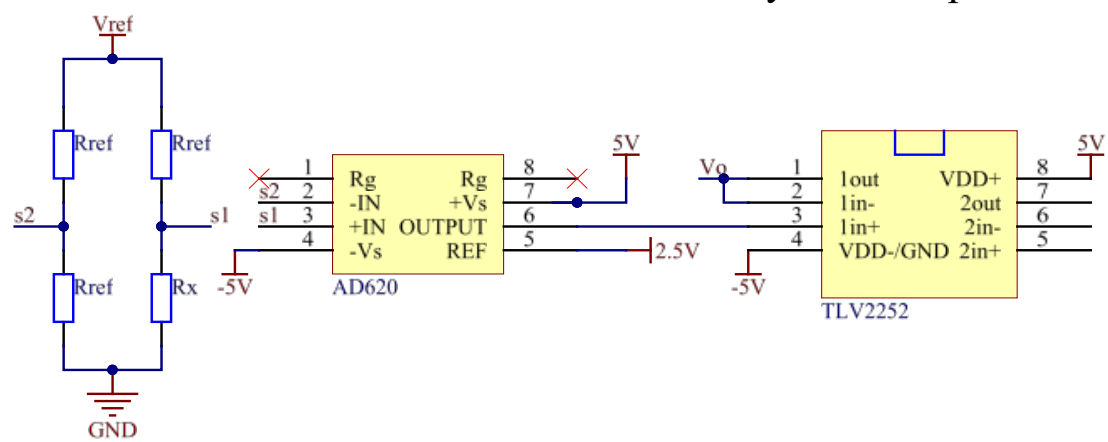

Fig. 4. Resistance measurement circuit 


\section{E. System Software}

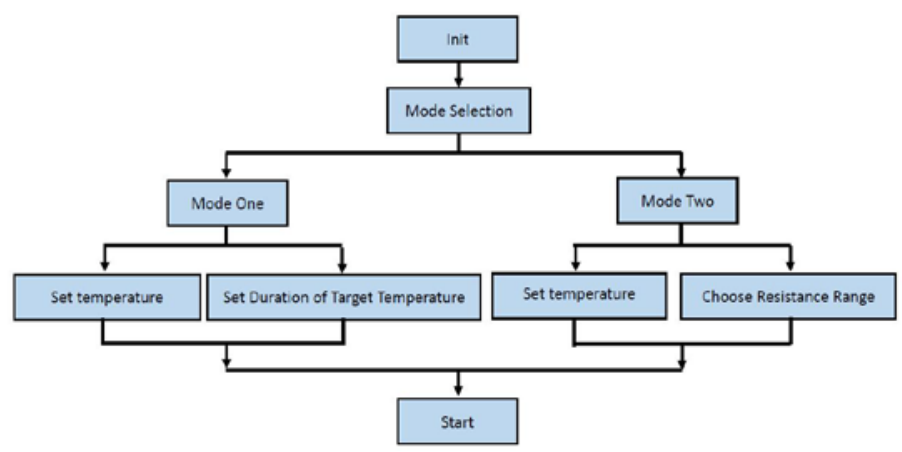

Fig. 5. Program design flowchart

The system has its own software including some of the operation, displaying temperature and resistance values of the curve and data exporting. Fig.5 shows the program design flowchart. First, the program is initialized and then choose work mode. If you choose one mode, step heating mode, you can set the temperature and the duration of the target temperature. The resistance bridge is judged by the ARM and selected through analog switch and the measurement range is $1 \Omega \sim 1 \mathrm{G} \Omega$. In another case, select the mode two. You can set a temperature and select the resistance range. Such as $200^{\circ} \mathrm{C}, 10 \Omega \sim 100 \mathrm{k} \Omega$. Finally, the test of the gas sensitive material starts to run.

\section{Experimental Results}

Table 1 shows the temperature testing results obtained by both the mercury thermometer and K-Thermocouple.

Table 1 temperature measurement data and measurement error

\begin{tabular}{|l|c|c|c|}
\hline & Actual temperature $\left({ }^{\circ} \mathrm{C}\right)$ & Measured temperature $\left({ }^{\circ} \mathrm{C}\right)$ & Absolute error $(\%)$ \\
\hline 1 & 23.6 & 23.6 & 0 \\
\hline 2 & 42.2 & 42.2 & 0 \\
\hline 3 & 61.3 & 61.4 & 0.16 \\
\hline 4 & 80.9 & 81 & 0.12 \\
\hline 5 & 102 & 102.7 & 0.68 \\
\hline 6 & 140.3 & 140.5 & 0.14 \\
\hline 7 & 184.6 & 186.4 & 0.97 \\
\hline 8 & 236.8 & 238.2 & 0.59 \\
\hline 9 & 259.8 & 260.2 & 0.15 \\
\hline 10 & 274.6 & 276.5 & 0.69 \\
\hline
\end{tabular}
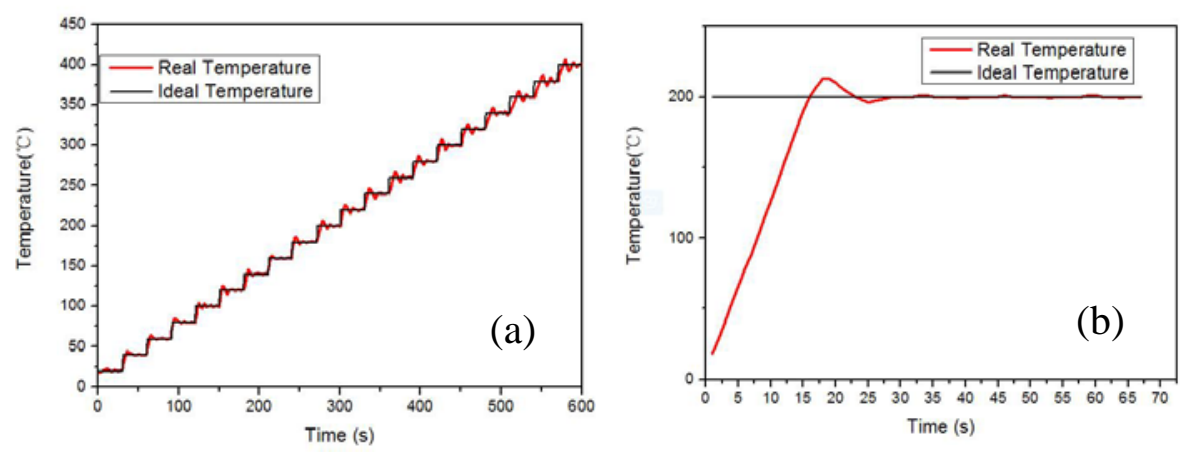

Fig. 6 Temperature curve: (a) mode 1 and (b) mode 2.

The absolute error of the measured temperature is less than 1\% and Fig. 6 shows the temperature curve of mode 1 and mode 2 . In particular, figure 6a) shows the result of a set temperature of 20, 40, $60,80 \ldots 400\left({ }^{\circ} \mathrm{C}\right)$, and the each target temperature duration is 30 s. Figure $\left.6 \mathrm{~b}\right)$ shows the result of a set temperature of $200^{\circ} \mathrm{C}$.

Table 2 shows the data of resistance measurements for 6 different measurement ranges. 
Table 2 resistance measurement data and measurement error

\begin{tabular}{|c|c|c|c|c|}
\hline Measurement ranges & Number & $\begin{array}{c}\text { Reference } \\
\text { resistance }(\Omega)\end{array}$ & $\begin{array}{l}\text { Measured } \\
\text { resistance }(\Omega)\end{array}$ & $\begin{array}{l}\text { Absolute error } \\
\text { (\%) }\end{array}$ \\
\hline \multirow{5}{*}{$1 \Omega \sim 10 \mathrm{k} \Omega$} & 1 & 1 & 1 & 0 \\
\hline & 2 & 10 & 9.88 & 1.2 \\
\hline & 3 & 100 & 100 & 0 \\
\hline & 4 & $1 \mathrm{k}$ & $0.99 \mathrm{k}$ & 1 \\
\hline & 5 & $10 \mathrm{k}$ & $9.9 \mathrm{k}$ & 1 \\
\hline \multirow{5}{*}{$10 \Omega \sim 100 \mathrm{k} \Omega$} & 1 & 10 & 9.98 & 0.2 \\
\hline & 2 & 100 & 99.5 & 0.5 \\
\hline & 3 & $1 \mathrm{k}$ & $1 \mathrm{k}$ & 0 \\
\hline & 4 & $10 \mathrm{k}$ & $9.87 \mathrm{k}$ & 1.3 \\
\hline & 5 & $100 \mathrm{k}$ & $99.7 \mathrm{k}$ & 0.3 \\
\hline \multirow{5}{*}{$100 \Omega \sim 1 \mathrm{M} \Omega$} & 1 & 100 & 98.5 & 1.5 \\
\hline & 2 & $1 \mathrm{k}$ & $0.98 \mathrm{k}$ & 2 \\
\hline & 3 & $10 \mathrm{k}$ & $10 \mathrm{k}$ & 0 \\
\hline & 4 & $100 \mathrm{k}$ & $99.8 \mathrm{k}$ & 0.2 \\
\hline & 5 & $1 \mathrm{M}$ & $0.98 \mathrm{M}$ & 2 \\
\hline \multirow{5}{*}{$1 \mathrm{k} \Omega \sim 10 \mathrm{M} \Omega$} & 1 & $1 \mathrm{k}$ & $0.98 \mathrm{k}$ & 2 \\
\hline & 2 & $10 \mathrm{k}$ & $10 \mathrm{k}$ & 0 \\
\hline & 3 & $100 \mathrm{k}$ & $100 \mathrm{k}$ & 0 \\
\hline & 4 & $1 \mathrm{M}$ & $0.98 \mathrm{M}$ & 2 \\
\hline & 5 & $10 \mathrm{M}$ & $9.87 \mathrm{M}$ & 1.3 \\
\hline \multirow{5}{*}{$10 \mathrm{k} \Omega \sim 100 \mathrm{M} \Omega$} & 1 & $10 \mathrm{k}$ & $9.89 \mathrm{k}$ & 1.1 \\
\hline & 2 & $100 \mathrm{k}$ & $99.8 \mathrm{k}$ & 0.2 \\
\hline & 3 & $1 \mathrm{M}$ & $1 \mathrm{M}$ & 0 \\
\hline & 4 & $10 \mathrm{M}$ & $9.8 \mathrm{M}$ & 2 \\
\hline & 5 & $100 \mathrm{M}$ & $98.9 \mathrm{M}$ & 1.1 \\
\hline \multirow{5}{*}{$100 \mathrm{k} \Omega \sim 1 \mathrm{G} \Omega$} & 1 & $100 \mathrm{k}$ & $98.9 \mathrm{k}$ & 1.1 \\
\hline & 2 & $1 \mathrm{M}$ & $0.98 \mathrm{M}$ & 2 \\
\hline & 3 & $10 \mathrm{M}$ & $10 \mathrm{M}$ & 0 \\
\hline & 4 & $100 \mathrm{M}$ & $99.8 \mathrm{M}$ & 0.2 \\
\hline & 5 & $200 \mathrm{M}$ & $197.9 \mathrm{M}$ & 1.05 \\
\hline
\end{tabular}

\section{Conclusions}

A novel measurement system for the characterization of gas sensing materials has been presented. Sheet shaped (unpackaged) gas sensitive material, especially brittle materials can be well measured by this test system. This system with two working modes is suitable for any phase of the study of gas sensing materials. Additionally, the system provides an accurate measurement of the sensing film temperature: the resolution is $0.1^{\circ} \mathrm{C}$ and the accuracy is up to $1 \%$. Temperature controlling is fast and stable by using the combination of conventional PID control and fuzzy PID control. The precision of the resistance measurement system is up to $2 \%$, and the test speed is 10 times per second even in the case of a large resistance (in mode two of one resistance range). This novel measurement system provides a possibility for the study of new gas sensing materials, and greatly improves the efficiency of materials research.

\section{Acknowledgement}

This work is financially supported by National Natural Science Foundation of China (Grant NO.61474056 and NO.61374218).

\section{References}

[1] Tommaso Addabbo, Francesco Bertocci, Ada Fort, Marco Mugnaini, Valerio Vignoli, Luay Shahin, Santina Rocchi, "Versatile Measurement System for the Characterization of Gas Sensing 
Materials”, Conference Record - IEEE Instrumentation and Measurement Technology Conference, p 976-980, 2013

[2] Ding Xibo, “Gas-sensor's Multiful Function Detective System”, Journal of Transducer Technology, vol. 16, no. 6, 1997, pp. 25-26.

[3] Guan GuoYu, Dai GuoRui, Gao Dingsan, “Design of Intelligent Gas Sensor Tester”, Chinese Journal of Scientific Instrument, vol. 18, no. 5, Oct. 1997, pp. 529-532.

[4] Cui Yuanhui, Tang Zhen’an, Yu Jun, Zhang Shuangyan, “Design of a Dynamic High-Precision Characterizing System for Gas Sensors”, Chinese Journal of Scientific Instrument, vol. 31, no. 10, Oct. 2010, pp. 2180-2185.

[5] A. Fort, S. Rocchi, M. B. Serrano-Santos, R. Spinicci, V. Vignoli, “Surface State Model for Conductance Responses During Thermal-Modulation of SnO2-Based Thick Film Sensors: Part I-Model Derivation”, IEEE Transaction on Instrumentation and Measurement, Vol. 55, n. 6, pp. 2102 - 2106, 2006.

[6] A. Fort, M. Mugnaini, S. Rocchi, M. B. Serrano-Santos, R. Spinicci, V. Vignoli, "Surface State Model for Conductance Responses During Thermal-Modulation of SnO2-Based Thick Film Sensors: Part II-Experimental Verification”, IEEE Transaction on Instrumentation and Measurement, Vol. 55, n. 6, pp. 2107 - 2117, 2006.

[7] Huang Yonghong, Zhu Mingxiang, Chen Zhaozhang, Xu Xiaobin, "Design of Precision Thermostat Bath Based on Fuzzy-PID Control”, Control Engineering of China, vol. 16, Sep. 2009, S3:80-82

[8] Wang Shuyan, Shi Yu, Feng Zhongxu, "A Method for Controlling a Loading System Based on a Fuzzy PID Controller”, Mechanical Science and Technology for Aerospace Engineering, vol. 30, no. 1, Jan. 2011, pp. 166-172. 